Tesis. Año 13, 12(15), 2019, 11-32

\title{
Una aproximación cuantitativa en la incidencia del uso de marcadores en la redacción de la prensa deportiva: el Torneo Clausura 2107 a través del diario El Bocón
}

\author{
Mark López Chumbe \\ marklopezchumbe@gmail.com
}

\section{Resumen}

Este artículo analiza la incidencia de los marcadores del discurso en la redacción de textos referidos al fútbol del diario deportivo El Bocón durante el Torneo Apertura 2017. Para ello, se registró y estudió un corpus de 104 textos del mencionado diario, lográndose los siguientes resultados: se verificaron 111 marcadores, 26 de ello se identificaron como innovadores, provenientes del habla coloquial peruano y cumplen la misma función propuesta por el inventario de Zorraquino \& Portolés (1999). Asimismo, se aprecia que el género textual del discurso deportivo de El Bocón para dicho torneo ha utilizado esencialmente los conectores y, en menor medida, los marcadores estructuradores y conversacionales. A partir de estos hallazgos, se infiere que muchos de estos marcadores discursivos resultan más apropiados para ciertos géneros textuales, en este caso para la redacción deportiva peruana.

Palabras claves: marcadores del discurso, conectores, contraargumentativos, periodismo deportivo, sensacionalismo, diario El Bocón.

\section{Abstract}

The objective of this paper is to analyze the incidence of speech markers in the football journalistic writing of sports newspaper El Bocón during the 2017 Apertura tournament. The corpus consisted of the analysis of 104 newspapers, achieving the following results: they verified a total of 111 markers, of which 26 were identified as innovators, since they come from the Peruvian colloquial speech and fulfill the same function proposed by the inventory of Zorraquino and Portolés (1999). Likewise, it can be seen that the textual genre of the sports speech of the El Bocón newspaper for the 2017 Apertura Tournament, has essentially used connectors and, to a lesser extent, structuring and conversational markers. From these findings it is inferred that many of these discursive markers are more appropriate for certain text genres, in this case for the Peruvian sports writing.

Keywords: speech markers, connectors, against argumentatives, sports journalism, sensationalism, El Bocón newspaper. 


\section{Una aproximación cuantitativa en la incidencia del uso de mareadores en la redaceión de la premsa deportiva: el Torneo Clausura 2107 a través del diario El Bocón}

\section{Introducción}

Los estudios sobre el uso de los marcadores discursivos en el ámbito periodístico - y principalmente deportivo - son muy escasos. Desde el plano de la comunicación social, a diferencia de la disciplina lingüística, existe un extensivo aporte sobre la relevancia de la comunicación escrita y — como afirma Pahuacho Portella (2017, p. 114) — gran parte del análisis y profundidad de la labor investigativa tiene como justificación la relevancia de la prensa en la construcción de la realidad del fútbol en el Perú.

Obviamente, existe una marcada tendencia por investigar este deporte desde la disciplina de la Comunicación Social. De los estudios que se han hecho en este rubro periodístico, el análisis de contenido de los corpus ha sido el más prolífico, teniendo como una de las líneas de investigación, identificar el mecanismo de articulación de los mensajes y cómo estos son transmitidos a los lectores.

Ahora, adentrándonos a la técnica de redacción, otro aspecto importante para rescatar son los numerosos cambios que ha sufrido al establecer nuevos cánones en la relación del idioma y los lenguajes que se emplean (Castañón Rodríguez \& Castañón Rodríguez 2006, p. 2). Este hallazgo no dejaría de ser singular a no ser por la preocupación inicial de este estudio: casi nada se ha hecho por definir, conceptualizar o analizar los marcadores del discurso. No obstante, es necesario, al menos, integrar algún modelo de análisis que nos permita conocer cómo y cuánto se escribe sobre el deporte en la actualidad.

Haciendo un resumido análisis situacional sobre la relevancia en la técnica de redacción deportiva, al igual que otra especialidad periodística, se adiciona un interés particular de "enganchar" a su público lector con noticias relevantes. En el Perú, esta situación particular no es ajena. Pese a la crisis funcional y estructural del fútbol profesional del país (exceptuando el triunfo de la selección peruana que, luego de 36 años, clasificó al Torneo Mundial de la FIFA celebrado en Rusia), la prensa deportiva, al parecer ha tenido que adaptarse para 
"sobrevivir" frente a un deporte carente de profesionalismo y que, no obstante, tiene una porción interesante de lectores que no solo gustan del fútbol local, sino también del internacional.

En concreto, uno de los remedios para atender una demanda especializada en la lectoría y persistir en el camino de informar sobre fútbol, ha sido la incorporación de técnicas sensacionalistas en la redacción periodística (Rojas Torrijos 2016, p. 49). Es decir, usar en las notas deportivas un lenguaje coloquial, muy informal, que incluye regularmente el rumor, el conflicto o las anécdotas como elementos imprescindibles. Ya no se escribe solo sobre los resultados o el ínterin de la contienda futbolística, también se analizan y exponen tópicos transversales o tangenciales de este deporte.

Por ejemplo, ahora, cuando se habla del mercado deportivo, se discute sobre las tentativas de compra de un futbolista talentoso a otros equipos, así solo sean simples especulaciones. Este fenómeno no es esencialmente nacional, por el contrario, al parecer, ha sido replicado en otros países con alto número de aficionados al fútbol, y tiene propósitos netamente comerciales. Esta espectacularidad del deporte rey — como afirma Sunkel (2002, p. 124) - es el canon que sigue la prensa deportiva sensacionalista: difundir un relato vivido con la capacidad de impresionar emocionalmente, utilizando una narrativa que mezcla las declaraciones recogidas de diferentes entrevistados, con la crónica.

Aunque no es objeto del presente estudio analizar la causa de estos cambios en la redacción, sino adentrarnos en el ámbito de los mensajes y procesos comunicacionales, la producción de los contenidos deportivos en las últimas décadas se fragua con base al avasallamiento de entretener e incrementar el éxito comercial; es decir, más ventas y publicidad para el diario, más que establecer puentes de responsabilidad social inherentes a la labor periodística, comprometerse a cambiar la coyuntura descrita u orientar al debate público sobre la verdadera situación de este deporte.

Dado el contexto expuesto, el objetivo de este trabajo se centra en analizar la incidencia de los marcadores del discurso en la redacción periodística del diario deportivo El Bocón durante el Torneo Apertura 2017. Para ello, se analizó el nivel de relevancia de los marcadores empleados en la redacción deportiva, así como se determinó su función y tratamiento en la técnica de redacción deportiva. Por lo tanto, este estudio busca otra mirada que, de por sí, procura no dejar de lado los procesos, estados y contextos que son necesarios para la emisión del mensaje, pues a través de la exploración de este estudio se conocieron varios de los aspectos discursivos y la relevancia que juega el uso de los marcadores.

Con respecto a la justificación de este trabajo, es importante valorar los hallazgos que contribuirán directamente a la enseñanza de las técnicas de redacción periodística. Además, con los aportes de esta investigación se podrán elaborar o proponer materiales y estrategias para mejorar el proceso de ense- 
ñanza de redacción general. Además, permitirá conocer el grado y relevancia de este aporte para la comprensión del fenómeno lingüístico en la rama comunicacional. La finalidad general consiste en desarrollar importantes contribuciones al uso de la lingüística en la redacción deportiva.

\section{Antecedentes}

Es importante advertir la casi ausencia de estudios sobre el tema tratado. Solo existe un estudio, al menos en el habla española, que involucra las variables que se pretenden analizar. El trabajo de Quintero Ramírez (2016, pp. 47- 69), bastante sui generis, tuvo como objetivo identificar los marcadores discursivos más recurrentes en un corpus de textos escritos por un comentarista deportivo. La relevancia del estudio reposa en la identificación de peculiaridades lingüísticas del discurso deportivo respecto de otros discursos. Con base a ello, los resultados más frecuentes fueron los marcadores llamados conectores, seguidos de los marcadores conversacionales.

También se revisaron algunos estudios sobre los diarios deportivos nacionales con la finalidad de tener conocimiento de las características de los medios deportivos impresos, influenciados por su público. No obstante, ninguna de estas investigaciones aborda el tema objeto de estudio, pero sí proporcionaron un panorama sobre el discurso deportivo en los medios impresos a nivel nacional.

Por ejemplo, el trabajo de Hernández (2012, p. 8) Criterios que rigen la producción de noticias sobre los fichajes de futbolistas profesionales en el Perú: el caso del diario Depor alcanzó las siguientes conclusiones: "Depor es un diario con un discurso verosímil y poco objetivo, pues pretende alcanzar una buena credibilidad con la audiencia deportiva, con el esparcimiento de rumores en el contenido de su sección impresa. Para lograrlo, el periodista estaría empleando un discurso fabulado y usaría varias técnicas (por ejemplo, estrategias discursivas) para hacer creer a la audiencia una hipotética realidad".

Morales (2012, p. 9) en su estudio titulado La información incompleta y limitada de los diarios especializados. Los casos de El Bocón, Libero y Todo Sport, sostuvo que "el contenido noticioso publicado en los tres diarios antes mencionados se enfoca básicamente en el fútbol, en perjuicio de los demás deportes practicados en el Perú. Según datos recogidos durante octubre de 2008, más del 64\% de su contenido es sobre noticias del balompié local y mundial”. Asimismo estos diarios se basan en la descripción de hechos, es decir, no existe análisis sobre la realidad del deporte peruano. Por otro lado, se aprecia un mínimo nivel de investigación en las noticias que fueron elaboradas por Libero y no se percibe un nivel avanzado de profundidad, pues se plantean muchas preguntas que no tienen nada que ver con la práctica deportiva. 
Por último, es importante incluir una atención especial respecto al uso de los marcadores del discurso empleados en el lenguaje juvenil, debido a la importancia e influencia en la redacción periodística deportiva. El estudio Myre \& Martínez (2007) es uno de los trabajos más representativos, pues indica que los jóvenes hacen un uso reducido de estas funciones por las características de la interacción de su propio lenguaje. Este estudio fue muy importante, pues ayudó a conocer si los marcadores del discurso empleados en las notas informativas de los diarios seleccionados provienen del habla juvenil.

\section{Tabla 1. Funciones usadas por los jóvenes de Madrid}

\begin{tabular}{|c|c|c|}
\hline \multicolumn{2}{|c|}{ Las funciones usadas por los jóvenes de Madrid } & N.० \\
\hline \multirow{3}{*}{$\begin{array}{l}\text { Estructuradores de la } \\
\text { información } 565\end{array}$} & Comentadores: pues (541), pues bien (2) & 543 \\
\hline & Ordenadores: por otro lado (3) & 3 \\
\hline & $\begin{array}{l}\text { Digresores: por cierto (13), a todo esto (4), a } \\
\text { propósito (2) }\end{array}$ & 19 \\
\hline \multirow{2}{*}{ Conectores 1136} & $\begin{array}{l}\text { Consecutivos: entonces (272), así pues (3), así } \\
\text { (310), pues (317), así que (54) }\end{array}$ & 956 \\
\hline & $\begin{array}{l}\text { Contra argumentativos: en cambio (4), sin em- } \\
\text { bargo (9) }\end{array}$ & 13 \\
\hline \multirow{4}{*}{ Reformuladores 605} & Explicativos: o sea (590) & 590 \\
\hline & De rectificación: más bien (3) & 3 \\
\hline & $\begin{array}{l}\text { De distanciamiento: en todo caso (3), de todos } \\
\text { modos (2) }\end{array}$ & 5 \\
\hline & Recapitulativos: en fin (7) & 7 \\
\hline \multirow{2}{*}{$\begin{array}{l}\text { Operadores argumenta- } \\
\text { tivos } 55\end{array}$} & $\begin{array}{l}\text { De refuerzo argumentativo: en realidad (8), en } \\
\text { el fondo (5), de hecho (2) }\end{array}$ & 15 \\
\hline & $\begin{array}{l}\text { De concreción: por ejemplo (39), en particular } \\
\text { (1) }\end{array}$ & 40 \\
\hline \multirow{4}{*}{$\begin{array}{l}\text { Marcadores Conversacio- } \\
\text { nales } 3723\end{array}$} & $\begin{array}{l}\text { De Modalidad epistémica: claro (324), desde } \\
\text { luego (13), por lo visto (8) }\end{array}$ & 345 \\
\hline & $\begin{array}{l}\text { De modalidad deóntica: bueno (80), bien (44), } \\
\text { vale (463) }\end{array}$ & 587 \\
\hline & $\begin{array}{l}\text { Enfocadores de la alteridad: hombre (136), oye } \\
\text { (209), mira (597) }\end{array}$ & 942 \\
\hline & $\begin{array}{l}\text { Meta discurso conversacionales: bueno (541), eh } \\
\text { (1085), e (223), este (10) }\end{array}$ & 1849 \\
\hline
\end{tabular}

Fuente: Myre y Martínez (2007). Los Marcadores del Discurso del Lenguaje Juvenil de Madrid. Nota: en este cuadro se puede observar que los conectores conversacionales "bueno" (541), "eh" (1085), "e" (223), "este" (10) los más empleados por los jóvenes madrileños en su hablar cotidiano, seguido del conector explicativo "o sea" (590.) 


\section{Los recursos literarios del periodismo deportivo en el Perú}

El periodismo deportivo, junto al económico, es muy especializado y generalmente ocupan las secciones habituales de cualquier medio de comunicación. Incluso se podría decir que se trata del más especializado dentro de los que cuentan con una difusión amplia (Paniagua, 2003). En el Perú, debido a su forma ágil y coloquial, este género periodístico es el que más tiraje y demanda tiene no solo en nuestro país, sino también en el mundo (Pahuacho Portella 2014, p. 153).

El alto nivel de lectoría de los diarios deportivos es importante. En el 2013, la Sociedad de Empresas Periodísticas del Perú, representaba el 21,3\% de la totalidad de diarios leídos semanalmente. El vínculo de continuidad que se logra establecer entre el lector y el diario tiene un rasgo notorio. Como indica Alcoba (2005, p. 137), los redactores deportivos "escriben como hablan" e integran el lenguaje vulgar o de la calle en sus textos. Al parecer, esta mezcla goza de aceptación social pues hace que la información deportiva sea más atractiva.

Agrandar los titulares es otra de las características en la redacción deportiva, así como la recurrencia a utilizar otros recursos literarios que, en gran parte de los casos, tergiversan la información y muchas veces se exageran los hechos. Esto, intencionalmente o no, lleva a consecuencias negativas. En algunos casos, se difunde información atentatoria contra el honor u honra de las personas sin mayor control ético. Además, se observa un descuido sobre lo que se difunde $y$, por ende, de crítica cuando se trata de situaciones injustas e incluso, lo que es más grave, la mentira oculta tras frases atribuidas a un emisor desconocido como "se ha dicho", "se comenta", "se rumorea insistentemente" (Vivanco 1992, p. 153). Otro tipo de recursos que se utilizan en la redacción deportiva suelen ser los siguientes:

El rumor: fuente de información infinita con dos caras. Se usa para sustituir información veraz si la demanda informativa se desborda y las fuentes oficiales no satisfacen las expectativas de los lectores. (Ritter 2000, p. 55)

El indicio de información: llamado también fuente indicativa provisional, es decir, señalador de una probabilidad noticiable sujeta a comprobación de otras fuentes. (Rivadeneira 1977, p. 74)

La magnificación de la información: consiste en la elaboración de múltiples variaciones para redactar una noticia. Por ejemplo, cuando un equipo le gana a otro, muchos periodistas refieren este hecho utilizando calificativos como superado, aplastado, barrido, humillado, acabado, aniquilado, y similares. (Hitchcock 1993, p.34)

Bajo estos parámetros de construcción, es bastante predecible que las crónicas, comentarios y reseñas se hayan convertido en un recurso muy utilizado para la elaboración de las notas informativas de los diarios deportivos. En 
suma, esta tratativa es una nueva manera de informar: evita los razonamientos y - provocado por las modernas tendencias del diseño en prensa deportivabusca una menor concentración de texto para proyectar un mayor atractivo a las páginas. Por lo mencionado, es fácil coincidir con Hernández (2012, p.42) que señala a la crónica como el género deportivo por excelencia por la audacia, el atrevimiento y peculiaridad del lenguaje deportivo.

\section{Los marcadores discursivos}

Para Zorraquino \& Portolés (1999, p. 4057) los marcadores "son unidades lingüísticas invariables. Además, poseen un cometido coincidente en el discurso, o sea orientar — de acuerdo con sus distintas propiedades morfosintácticas, semánticas y pragmáticas - las inferencias que suceden en la comunicación”. Sus principales funciones son las siguientes: visibilizan las relaciones estructurales del contenido; apoyan la localización de la información; brindan fuerza, cohesión y aseguran la continuidad del discurso.

$\mathrm{El}$ adecuado uso de un marcador dependerá no solo de las propiedades gramaticales, sino del esfuerzo por lograr la comprensión textual: obligando al lector, a desarrollar las inferencias de un modo determinado. Esto se debe a que los hablantes se comunican representando un estímulo que permite al oyente conseguir, por medio de inferencias, lo que se pretende comunicar (Zorraquino \& Portolés, 1999, p. 4058). Este afianzamiento interactivo favorece el cambio del tema de la comunicación y el uso de expresiones que indican que el hablante ha recibido el mensaje emitido por el oyente, o que ha comprendido dicho mensaje, o que desea mantener el contacto comunicativo (Zorraquino \& Portolés 1999, p. 4143).

Los marcadores discursivos también gozan de diferentes acepciones. Díaz (1987) los denomina 'conectores' por su función: orientar al lector en el descubrimiento del texto y la organización de la información. Además, resalta la característica de no conmutabilidad de algunos 'conectores', pues la gran mayoría de ellos son polisémicos. En cambio - para Murillo (2007) - los marcadores portan sus propias funciones y por sus diferencias, tienden a distinguirse entre subgrupos y elementos, y poseen varias características fundamentales, tales como: i. Significado procedimental ii. Rol de guías de inferencias iii. Rol de organizadores de la estructura discursiva. Haciendo un resumen, también pueden ser llamados "conectores discursivos/del discurso" (Portolés 1995) (Montolío 1992); conectores pragmáticos (Fuentes 1993), entre otros.

Con respecto a su clasificación, utilizaremos la propuesta de Zorraquino \& Portolés (1999, p. 4056) y algunas advertencias fijadas por ambos autores: los marcadores del discurso son difíciles de sistematizar y su propuesta no es un todo uniforme. Sin embargo, en un intento por juntarlos se distinguen, cinco grupos: 


\section{Tabla 2. Clasificación de Marcadores del discurso}

\begin{tabular}{|c|c|}
\hline Marcadores & Tipos \\
\hline \multirow{11}{*}{$\begin{array}{l}\text { Estructuradores } \\
\text { de la informa- } \\
\text { ción }\end{array}$} & $\begin{array}{l}\text { Comentadores: Incluyen un nuevo comentario ("pues", "pues bien”, } \\
\text { "así las cosas") }\end{array}$ \\
\hline & Ordenadores: Está conformado por varias secciones del discurso \\
\hline & - Enumeración. "primero" "segundo" "tercero" \\
\hline & - Espacial: "por un lado" "por otro lado" "por una parte" "por otra \\
\hline & parte". \\
\hline & - Temporal: “después”, “luego”, “finalmente”. \\
\hline & $\begin{array}{l}\text { - Pares correlativos: "por un lado/por otro (lado)", "por una parte/ } \\
\text { por otra (parte)", entre otros. En algunos casos, pueden estar se- } \\
\text { guidos por un tercer marcador de tipo ordenador. Este subtipo de } \\
\text { ordenadores se clasifica en tres sub sub tipos: }\end{array}$ \\
\hline & $\begin{array}{l}\text { Marcadores de apertura: "en primer lugar", "primeramente", } \\
\text { "por un lado". Abren una secuencia en el discurso. }\end{array}$ \\
\hline & $\begin{array}{l}\text { Marcadores de continuidad: "en segundo/tercer/.../ lugar", } \\
\text { "por otra parte", "por otro lado". El miembro que acompaña } \\
\text { forma parte de una serie de la cual no el elemento inicial. }\end{array}$ \\
\hline & $\begin{array}{l}\text { Marcadores de cierre: "por último", "en último lugar", "final- } \\
\text { mente". Es el fin de una serie discursiva. }\end{array}$ \\
\hline & $\begin{array}{l}\text { Digresores: "por cierto", "a todo esto", "a propósito". Introducen un } \\
\text { comentario lateral respecto de la planificación del discurso anterior. }\end{array}$ \\
\hline \multirow{4}{*}{ Conectores } & $\begin{array}{l}\text { Conectores aditivos: Unen a un miembro anterior con otro de su } \\
\text { misma orientación ("además", "encima", "aparte", "incluso"). }\end{array}$ \\
\hline & $\begin{array}{l}\text { Conectores consecutivos: Conectan a un consecuente con su an- } \\
\text { tecedente ("por tanto", "por consiguiente", "por ende", "en conse- } \\
\text { cuencia"). }\end{array}$ \\
\hline & $\begin{array}{l}\text { Conectores Causales: Ponen de relieve la causa en la relación de } \\
\text { causa-efecto ("a causa de", "porque", "por ello", "puesto que”, "ya } \\
\text { que", "dado que") }\end{array}$ \\
\hline & $\begin{array}{l}\text { Conectores contra argumentativos: Eliminan algunas de las con- } \\
\text { clusiones que pudieran inferirse de un miembro anterior ("en cam- } \\
\text { bio", "por el contrario", "sin embargo", "no obstante"). }\end{array}$ \\
\hline \multirow{4}{*}{ Reformuladores } & $\begin{array}{l}\text { Explicativos: Presentan un nuevo miembro del discurso como una } \\
\text { explicación anterior ("o sea", "esto es”, "es decir"). }\end{array}$ \\
\hline & $\begin{array}{l}\text { Rectificativos: Corrigen a un miembro discursivo anterior ("mejor } \\
\text { dicho", "mejor aún", "más bien”). }\end{array}$ \\
\hline & $\begin{array}{l}\text { Distanciamiento: Privan de pertinencia al miembro discursivo an- } \\
\text { terior ("en cualquier caso", "en todo caso", "de todos modos"). }\end{array}$ \\
\hline & $\begin{array}{l}\text { Recapitulativos: Introducen una recapitulación o conclusión de un } \\
\text { miembro discursivo anterior o una serie de ellos (“en suma”, "en } \\
\text { conclusión”, "en definitiva”, “en fin”, "al fin y al cabo"). }\end{array}$ \\
\hline
\end{tabular}




\begin{tabular}{|c|c|}
\hline $\begin{array}{l}\text { Operadores } \\
\text { argumentativos }\end{array}$ & $\begin{array}{l}\text { a. Operadores de refuerzo argumentativo: Su significado refuerza } \\
\text { como argumento el miembro del discurso en el que se encuentra } \\
\text { frente a otros posibles argumentos ("en realidad", "en el fondo", } \\
\text { "de hecho"). } \\
\text { b. Operadores de concreción: Muestran el miembro del discurso } \\
\text { en el que se localizan como una concreción o un ejemplo de una } \\
\text { generalización ("por ejemplo", "en particular"). }\end{array}$ \\
\hline $\begin{array}{l}\text { Conversacio- } \\
\text { nales }\end{array}$ & $\begin{array}{l}\text { Aquellos que se utilizan frecuentemente en la conversación, y que, } \\
\text { además, se caracterizan por cierta autonomía, pues pueden apa- } \\
\text { recer solos en una intervención. (Martín Zorraquino, 1999). Los } \\
\text { marcadores conversacionales se distribuyen en cuatro grupos: } \\
\text { a. De modalidad epistémica: Señalan el grado de certeza, de evi- } \\
\text { dencia, que el hablante atribuye al miembro o a los miembros } \\
\text { del discurso con el que se vincula cada partícula ("claro", "por lo } \\
\text { visto", "desde luego"). } \\
\text { b.De modalidad deóntica: Indican diversas actitudes volitivas del } \\
\text { hablante respecto del miembro o miembros del discurso en el que } \\
\text { aquellos comparecen ("bueno", "bien vale"). } \\
\text { c. Enfocadores de la alteridad: Orientan sobre la forma como el } \\
\text { hablante se sitúa en relación con su interlocutor en la interacción } \\
\text { comunicativa ("hombre", "mira", "oye"). }\end{array}$ \\
\hline $\begin{array}{l}\text { Meta discursivos } \\
\text { conversacionales }\end{array}$ & $\begin{array}{l}\text { Sirven para estructurar la conversación; es decir, para distinguir } \\
\text { bloques informativos, por ejemplo, o para alternar o mantener los } \\
\text { turnos de palabra, etcétera ("bueno", "eh", "este"). }\end{array}$ \\
\hline
\end{tabular}

Fuente: Zorraquino \& Portolés (1999, p. 4056)

Zorraquino \& Portolés (1999, p. 4081) sostuvieron la relación de los conectores con la semántica, debido a que los marcadores no poseen un valor sémico; es decir, no tienen un significado semántico designativo, más bien sus elementos poseen un significado de procesamiento sobre el contenido de los enunciados que se enlazan. Por tanto, el adecuado uso del marcador dependería de las propiedades gramaticales y, con una mayor importancia, del esfuerzo para lograr la comprensión del discurso. Los conectores no se conciben como nexos que conectan una oración o proposiciones con otras, más bien identifican la unidad que está formada por una serie de instrucciones que hace comprender la relación semántica entre los miembros conectados, pero desde una forma predeterminada (Portolés, 1998, p. 72).

Por otro lado, Camps (1976, p. 50) identifica la relación pragmática de los marcadores como un lenguaje viable. La forma en el uso del lenguaje trasciende su aplicación teórica y cumple su labor destinada: que es de la función comunicativa. Obviamente, una palabra o un nombre carecen de poder comunicativo, es decir, si no existe un contexto para definir el acto lingüístico. De esta ma- 
nera, la pragmática cumple la labor de identificar y reconstruir las condiciones universales de toda comprensión posible (Habermas 1987, p. 174). Un ejemplo interesante de esta teoría es el propuesto por Nogueira (2010):

\section{Lula es inteligente $y$, sin embargo, pobre.}

Como señala el autor, el contenido semántico del marcador sin embargo no afecta a las condiciones de verdad que deben satisfacer las proposiciones: i. Lula es inteligente y ii. Lula es pobre. Puesto que el papel de este conector es indicar que ambos contenidos deben ser procesados como contradictorios, a partir de esta inferencia se entiende que los marcadores no estarían contribuyendo directamente al significado conceptual de las intervenciones ni a sus condiciones de verdad, pero sí a su procesamiento, esto es, a la realización de unas inferencias determinadas a partir de la relación de lo dicho y el contexto (Portolés 1998, p. 22).

Ducrot \& Anscombre (1994, p. 25), señalan la idea de que el lenguaje no es primordialmente denotativo; pues esto les lleva a defender una semántica no veritativa (por eso rechazaron el verificacionalismo) que es la base de la teoría de la argumentación, sintagmática y no opuesta a la pragmática.

La fonología suprasegmental pone especial atención en la prosodia, debido a que, en ocasiones, consiste en un recurso demarcativo determinante para la segmentación de discurso en actos (Hidalgo Navarro 1997). En este caso, se presta atención a las siguientes marcas prosódicas: pausa, presencia de una curva melódica completa o el empleo de una entonación final marcada en los enunciados aseverativos (con un tonema ascendente $[\uparrow]$ o descendente $[\downarrow]$ ). Estos elementos son imprescindibles de la estructura gramatical como acto.

\section{Metodología}

\subsection{Constitución del corpus}

Para el presente estudio hemos considerado 104 ediciones del diario El Bocón durante todo el Torneo Clausura 2017, el cual empezó el 17 de agosto y terminó el 3 de diciembre de 2017. Los textos analizados solo se centran en el acontecer deportivo del torneo y se han excluido otros tópicos, tales como fútbol internacional, otros torneos nacionales y noticias de otras disciplinas deportivas. Por otra parte, es importante señalar que hemos optado por analizar los marcadores discursivos que, en posteriores estudios, podrían extrapolarse en relación con otros diarios o periodistas deportivos. 


\subsection{Procedimiento de análisis}

Luego de caracterizar los textos que constituyen el corpus de esta investigación, se procedió a clasificar los marcadores discursivos que se han registrado, llevándose a cabo un análisis descriptivo con el apoyo de una metodología cuantitativa. En primer lugar, se elaboró una lista de marcadores verificados en la propuesta de Zorraquino \& Portolés (1999, p. 4056) y que conforman nuestro apartado teórico. Por otra parte, para mayor facilidad y hacer mucho más eficiente el reconocimiento de los marcadores, los periódicos utilizados fueron de formato electrónico tipo Portable Document Format (PDF).

Luego, para elaborar una línea de base operacional - como se observa en la Tabla - se registraron un total de 111 tipos de marcadores, de los cuales un 61.26\% fueron del tipo conector, un $14.41 \%$ fueron del tipo estructurador, un $11.71 \%$ son del tipo reformuladores, mientras que un $8.11 \%$ fueron conversacionales y $4.5 \%$ fueron del tipo argumentativos.

\section{Diario El Bocón-Torneo apertura: tipo de Marcadores del discurso (en frecuencia y \%), 2017}

\begin{tabular}{|l|c|c|}
\hline \multicolumn{1}{|c|}{ Marcadores del discursos } & $\begin{array}{c}\text { Tipos de } \\
\text { marcadores }\end{array}$ & \% \\
\hline Conectores & 68 & 61.26 \\
\hline Estructuradores de la información & 16 & 14.41 \\
\hline Reformuladores & 13 & 11.71 \\
\hline Marcadores conversacionales & 9 & 8.11 \\
\hline Operadores argumentativos & 5 & 4.5 \\
\hline Total & 111 & 100 \\
\hline
\end{tabular}

Elaboración: propia

Es importante añadir que al momento de localizar estos marcadores, algunos no registraron ningún tipo de frecuencia. Esto quiere decir que no fueron hallados en los textos analizados, lo que podría deberse a que han caído en desuso. Por tanto, se ha decidido presentar en esta clasificación los marcadores discursivos que sí fueron localizados en el corpus.

Estos marcadores identificados fueron previamente localizados en un programa de análisis cuantitativo llamado Atlas Ti que, a su vez, almacenó los periódicos en formato PDF. De esta manera, fueron clasificados y agrupados. Posteriormente, se utilizó una distribución sistemática de datos relevantes que 
posibilitaron una mejor comprensión del objeto de estudio. Por último, se elaboraron cuadros estadísticos descriptivos tales como frecuencia y porcentajes para los resultados y su posterior análisis.

Por último, es importante señalar que también se identificaron a 26 marcadores que no forman parte del inventario propuesto por Zorraquino \& Portolés (1999), pero que dentro del proceso de verificación desarrollan atributos y funcionalidades propias de la categorías propuesta por ambos autores. Este grupo de marcadores identificados se les ha denominado "innovadores", pues vienen del habla coloquial peruana y también buscan establecer cierta relación de familiaridad de los lectores de este tipo de prensa. Para el proceso de verificación y recurrencia se identificaron a los siguientes:

\section{Conectores}

Aditivos: $y$ también, por lo demás, y después, lo mismo, encima, es más

Causales: si es que, es que, y es que

Consecutivos: y luego, ante eso, es por eso que, y eso, y asi

Contra argumentativos: en seguida, y si bien, y aunque, es asi que, y abora, por el momento, fue asi que, por ahora, eso si, esta vez, si bien

\section{Reformuladores}

Explicativos: De igual modo

\section{Marcadores conversacionales}

De modalidad epistémica: claro y sin $d u d a$

\section{Análisis y resultados}

De acuerdo al procesamiento de los resultados del estudio, se observa que fueron recogidos un total de 7889 marcadores en total, de los cuales - como se observa en el Gráfico 1 -, un $72.86 \%$ de los marcadores son de tipo conectores, seguido, aunque con una minoritaria participación los estructuradores (15.97\%), conversacionales (8.24\%), reformuladores (2.37\%) y argumentativos $(0.58 \%)$, respectivamente. 


\section{Diario El Bocón- Torneo Apertura 2017: Marcadores del discurso totales (en \%)}

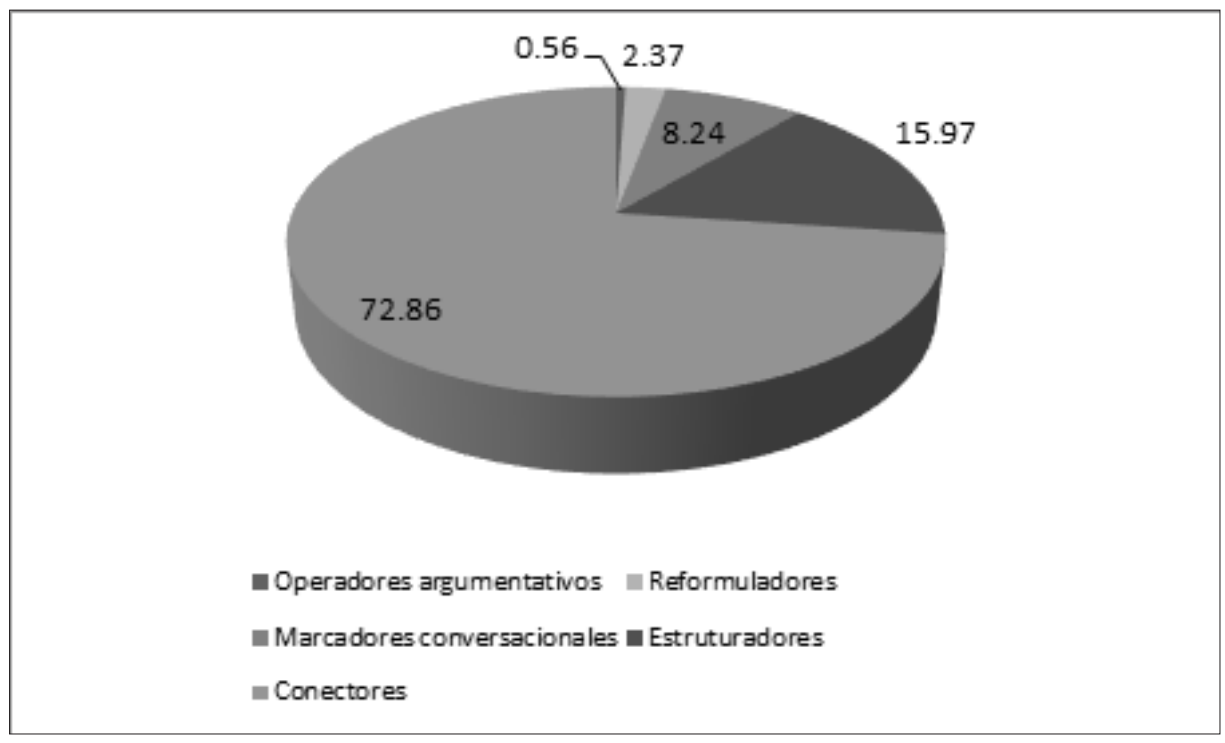

Elaboración: propia

Si bien este resultado nos inclina a desarrollar una lectura con mayor profundidad a los marcadores del tipo conector, también se profundizarán las otras categorías.

\subsection{Conectores}

Ahora bien, con respecto a los marcadores conectores, se aprecia que un 42.92\% son contraargumentativos, seguidos de los consecutivos (22.74\%), aditivos (21.99\%) y causales (12.35\%). La cantidad de marcadores de la subcategoría aditivos presentó 17 marcadores cuya frecuencia registrada suma un total de 1264 marcadores, donde encontramos a "además" con un 45.8\%, seguido, aunque en menor proporción, de "después" (12.82\%) y "asimismo" (9.57\%) (Anexo 1).

Por otra parte, también se identifican una serie de marcadores innovadores, aunque usados con menor frecuencia. Estos son los siguientes: "y también" (0.16\%), "por lo demás”, (0.24\%) “y después” (0.32\%), "lo mismo” (1.11\%), "encima” (2.53\%) y "es más" (4.27\%).

Con respecto a la subcategoría causales, se identificaron un total de 9 tipos de marcadores: "por eso" representó el 33.8\%, seguido del marcador "por ello" con un $30.28 \%$. También se registraron marcadores innovadores en esta categoría, siendo el más representativo el marcador "y es que" con un 23.52\%, 
seguido, aunque en menor medida, por el marcador "es que" con un 4.08\% y "si es que" con un $0.85 \%$ respectivamente (Anexo 2 ).

Ahora bien, con respecto a la subcategoría de consecutivos, se aprecia que el marcador "luego" concentra el 36.88\% del total de utilizados, seguido de "así" con un $30.83 \%$, "así que" con un $15.23 \%$. Aunque con una proporción menor al $3 \%$, también se identifican algunos marcadores innovadores, tales como "y así", "y eso", "es por eso que", "ante eso", "y luego" (Anexo 3).

Como se mencionó anteriormente, los conectores contraargumentativos son los más empleados en esta categoría. se identificaron a 21 tipos, siendo el más utilizado el marcador "sin embargo" con un $26.83 \%$, seguido por "pero" con un $22.7 \%$ y "ahora" con un $16.3 \%$. En esta subcategoría es importante identificar que se recogen una variedad de marcadores innovadores. Entre ellos, el más recurrente fue el marcador "si bien" con un 5.35\%. Los otros marcadores identificados como innovadores son los siguientes: "esta vez", "eso sí”, "por ahora", "fue así que", "por el momento", "y ahora", "es así que", "y si bien" y "en seguida”. (Anexo 4.)

\subsection{Reformuladores}

Con respecto a esta categoría, solo se identificaron a 13 tipos de marcadores utilizados, pero con respecto a su frecuencia de uso, se identifica que casi todos los marcadores verificados pertenecieron a la subcategoría explicativos (85.03\%). El resto de subcategorías, tales como rectificativos, de distanciamiento y recapitulativos tiene una menor participación porcentual $(4.38 \%, 8.56 \% \mathrm{y}$ $2.14 \%$ respectivamente).

Sobre el uso de marcadores explicativos, el más frecuente fue el uso del marcador "esto es", (34.59\%) seguido de "igual" (25.79\%), "es decir" (22.64\%), "o sea" (12.58\%) y "de igual modo" (4.4\%); siendo este último identificado como marcador innovador (Anexo 5.). Con respecto a la subcategoría rectificativa, solo se identificó al marcador “más bien”, el cual solo se utilizó 8 veces (Anexo 6).

Sobre la subcategoría de distanciamiento, se observa que el más utilizado fue el marcador "de todos modos" con un 56.25\%, seguido de "de todas maneras" con un 25\% (Anexo 6). Por último, en los marcadores recapitulativos se verifica: "en conclusión” y "en fin" (Anexo 7).

\subsection{Estructuradores de la información}

Con respecto a esta categoría, solo se identificaron a 16 tipos de marcadores utilizados. Asimismo, se aprecia que gran parte de los marcadores verificados correspondían a la subcategoría de ordenadores con $83.3 \%$, seguidos de comentadores $(15.87 \%)$ y, aunque con una menor proporción, los digresores $(0.79 \%)$. 
Ahora bien, con respecto a la subcategoría de comentadores, se aprecia que el marcador "por otro lado" es el más utilizado por excelencia (anexo 9). También se identifican otro tipo de marcadores, tales como "pues", "pues bien" y "así las cosas", pero no representan más de $6 \%$.

Con respecto a la subcategoría de digresores solo se aprecia el uso del marcador "a propósito" (Anexo 10). En la subcategoría ordenadores se concentran los marcadores de mayor uso por los redactores periodísticos. un $45.9 \%$ corresponde al marcador "luego", seguido del marcador "finalmente" (1.73\%), "después” (15.4\%), “de otro lado" (7.8\%), entre otros (Anexo 11).

\subsection{Operadores argumentativos}

En esta subsección solo se identificaron a 5 tipos de marcadores utilizados, además la recurrencia del uso de esta categoría es mucho menor que las anteriores (44 marcadores verificados), tal como se explica en la sección 4. Con respecto a la subcategoría de refuerzo argumentativo, se aprecia que representó un 65.71\%, mientras que el de concreción alcanzó un 34.09\% (Anexo 12 y Anexo 13 , respectivamente).

\subsection{Marcadores conversacionales}

Por último, con respecto a esta categoría solo se identificaron a 9 tipos de marcadores, siendo la recurrencia de la subcategoría de enfocadores de la alteridad la más utilizadas, seguida a los de modalidad epistémica (35.58\%) y deóntica (17.48\%).

Con respecto a la subcategoría epistémica, se aprecia que dos marcadores innovadores fueron los más representativos: "claro" (30.17\%) y "sin duda" (47.41\%) (Anexo 14). En los de modalidad deóntica solo se identifica la recurrencia del marcador "bien" (Anexo 15), mientras que los enfocadores de la realidad solo se identificó el marcador "bueno" (Anexo 16).

\section{Conclusiones}

Se recogieron un total de 7889 marcadores, los cuales un $72.86 \%$ de ellos son de tipo conectores, seguido, aunque con una minoritaria participación, los estructuradores (15.97\%), conversacionales (8.24\%), reformuladores $(2.37 \%)$ y argumentativos $(0.58 \%)$ respectivamente.

Los conectores del subtipo contraargumentativos muestran una preferencia regular con respecto a su uso. Se observa que el marcador "sin embargo" es el más utilizado con un $26.83 \%$, luego "pero" con un $22.7 \%$ y "ahora" con un $16.3 \%$. Con ello, se infiere que muchos redactores deportivos prefieren redactar sus notas, no sin antes establecer algunas situaciones no necesariamente 
contradictorias, pero que resultan atrayentes para "enganchar al lector". En el caso de los marcadores reformuladores, se aprecia que la subcategoría explicativos fue la más empleada (85.03\%). "De todos modos" con un 56.25\%, seguido de "de todas maneras" con un $25 \%$ fueron los más utilizados por esta categoría.

Con respecto a la categoría de estructuradores de la información, casi todos fueron de la categoría de ordenadores, siendo el marcador "luego" el más utilizado para explicar los hechos que va narrando el lector.

Ahora bien, el género textual del discurso deportivo del diario El Bocón para el Torneo Apertura 2017 ha utilizado esencialmente los marcadores del tipo conectores y, en menor medida, los marcadores estructuradores y conversacionales. A partir de estos hallazgos se infiere que muchos de estos marcadores discursivos resultan más apropiados para ciertos géneros textuales, en este caso para la redacción periodística deportiva.

Por otra parte, también se identifica que la frecuencia del uso de marcadores innovadores son de la categoría conectores; siendo los más utilizados "y es que”, “si bien”, “esta vez”, “eso sî”, "es más”, “y así”, “y eso”, entre otros. Como se aprecia, los redactores introducen marcadores de habla coloquial con la finalidad de arraigar sus discursos con elementos del hablan común peruana.

Este trabajo aspira a contribuir al análisis del discurso deportivo desde los marcadores discursivos. La exploración realizada evidencia la necesidad de continuar en el estudio de los elementos lingüísticos en la producción de textos periodísticos. En este caso, los textos deportivos constituyen un corpus interesante y provisto de una mezcla mayoritaria de marcadores del tipo contraargumentativos. En esencia, se observa que la objetividad de las notas periodística puede estar sujeta a premisas contradictorias que no solo avalan lo mencionado, sino agregan ciertas pautas de incertidumbres con la finalidad de atraer y fidelizar al público lector.

\section{Referencias bibliográficas}

Alcoba, A. (2005). Periodismo deportivo. Madrid: Síntesis.

Anscombre, J. \& Ducrot, O. (1994). La argumentación en la lengua, versión española de J. Sevilla \& M. Tordesillas, introducción de M. Tordesillas. Madrid: Gredos.

Camps, V. (1976). Pragmática del leguaje y filosofía analítica. Barcelona: Península.

Castañón R., M. \& Castañón Rodríguez,J. (2006) Cambios en la redacción periodística del deporte en América y España en el Siglo XXI. Revista Digital Universitaria. Volumen 7 número 6. México D.F.: Universidad Autónoma de México, 1- 10

Díaz, Á. (1987). Aproximación al texto escrito. Medellín. Universidad de Antioquia. 
Hidalgo, A. (1997). Entonación coloquial. Función Demarcativa y Unidades de Habla. Valencia. Anejo XXI de la revista Quaderns de Filologia. Universitat de València

Habermas, J. (1987b). Teoría de la acción comunicativa. Volumen 1: Racionalidad de la acción y racionalización social. Madrid. Taurus.

Hitchcock, J. R. (1993). Periodismo deportivo. Santa Fe de Bogotá: Voluntad.

Hernández Castillo, R. (2012). Criterios que rigen la producción de noticias sobre los fichajes de futbolistas profesionales en el Perú: el caso del diario Depor (Tesis de Licenciatura). PUCP, Lima.

Jørgensen, A. M., López, J. \& Martínez, A. (2007). Los marcadores del discurso del lenguaje juvenil de Madrid. Revista Virtual de Estudos da Linguagem- ReVEL. Vol. 5, n. 9, agosto de 2007. ISSN 1678-8931 [www.revel.inf.br].

Nogueira da Silva, A. M. (2010) Las teorías pragmáticas y los marcadores del discurso. Revista de Divulgação Científica em Lingua Portuguesa, Lingüística e Literatura. Año 06, n.13- 2o Semestre de 2010.

Morales Rodríguez, F. A. (2012). Información incompleta y limitada de los diarios especializados Los casos de El Bocón, Libero y Todo Sport (Tesis de Licenciatura). Lima. UNMSM.

Zorraquino, M. \& Portolés, J. (1999). Los marcadores del discurso. En Bosque, I. y Demonte, V. (eds.) Gramática Descriptiva de la Lengua Española (pp. 4051- 4213) Madrid: Espasa-Calpe.

Pahuacho Portella, A. (2014). El tópico sacrificial en los discursos de la prensa deportiva en el Perú. El caso del futbolista Paolo Guerrero. Revista Correspondencias E̊ Análisis, No 4. Lima, Pontificia Universidad Católica del Perú.

Paniagua Santamaría, P. (2003). La crítica y el deporte: el placer de la influencia". Estudios sobre el Mensaje Periodístico. Madrid: Servicio de Publicaciones de la Universidad Complutense.

Portolés, J. (1998). La teoría de la argumentación en la lengua y los marcadores del discurso. en Martín Zorraquino, M. A. y Monotolío Durán, E. (eds.). Los marcadores del discurso: teoría y análisis. Madrid: Arco/Libros, 71- 92

Quintero Ramírez, S. (2016). Marcadores discursivos en el blog de José Ramón Fernandez. Lingüística y Literatura número 70. Guadalajara. Universidad de Guadalajara, 47- 69.

Roja Torrijos, J. L. (2016). La creciente banalización de los contenidos deportivos. Cuadernos de periodistas número 31. Madrid: asociación de la Prensa de Madrid. pp. 48- 56 Recuperado de http://www.cuadernosdeperiodistas.com/pdf/Cuadernos_de_Periodistas_31.pdf

Sunkel, G. (2002). La prensa sensacionalista y los sectores populares. Bogotá. Norma.

Vivanco, Á. (1992). Los libertadores de opinión y de información. Chile. Andrés Bello. 
Ritter, M. (2000) El rumor: un análisis epistemológico. Ritters \& Partners Comunicación Estratégica. Buenos Aires, Argentina. Recuperado de: http://ritterandpartners.medianwebstudio.de/es/documentos/El_rumor_Un_anlisis_epistemolgico.pdf

\section{Anexos}

Anexo 1. Conectores - Aditivos

\begin{tabular}{|l|r|r|}
\hline Marcadores & \multicolumn{1}{|c|}{$\mathbf{N}^{\circ}$} & \multicolumn{1}{c|}{$\mathbf{\%}$} \\
\hline Y también & 2 & 0.16 \\
\hline Cabe señalar que & 3 & 0.24 \\
\hline Por lo demás & 3 & 0.24 \\
\hline Y después & 4 & 0.32 \\
\hline Y después & 4 & 0.32 \\
\hline Poco después & 4 & 0.32 \\
\hline Lo mismo & 14 & 1.11 \\
\hline Aparte & 15 & 1.19 \\
\hline Más aún & 29 & 2.29 \\
\hline Encima & 32 & 2.53 \\
\hline Es más & 54 & 4.27 \\
\hline Incluso & 55 & 4.35 \\
\hline También & 91 & 7.20 \\
\hline Por su parte & 92 & 7.28 \\
\hline Asimismo & 121 & 9.57 \\
\hline Después & 162 & 12.82 \\
\hline Además & 579 & 45.81 \\
\hline Total & 1264 & 100.00 \\
\hline
\end{tabular}

Anexo 2. Conectores - Causales

\begin{tabular}{|l|r|r|}
\hline Marcadores & \multicolumn{1}{|c|}{$\mathbf{N}^{\circ}$} & \multicolumn{1}{c|}{$\mathbf{\%}$} \\
\hline A causa de & 1 & 0.14 \\
\hline Puesto que & 1 & 0.14 \\
\hline Si es que & 6 & 0.85 \\
\hline Ya que & 7 & 0.99 \\
\hline Es que & 29 & 4.08 \\
\hline Porque & 44 & 6.20 \\
\hline Y es que & 167 & 23.52 \\
\hline Por ello & 215 & 30.28 \\
\hline Por eso & 240 & 33.80 \\
\hline Total & 710 & 100.00 \\
\hline
\end{tabular}


Anexo 3. Conectores - Consecutivos

\begin{tabular}{|l|r|r|}
\hline Marcadores & \multicolumn{1}{|c|}{$\mathbf{N}^{\circ}$} & \multicolumn{1}{|c|}{$\%$} \\
\hline En consecuencia & 1 & 0.08 \\
\hline A partir de ello & 1 & 0.08 \\
\hline Por tanto & 3 & 0.23 \\
\hline Y luego & 4 & 0.31 \\
\hline De lo contrario & 5 & 0.38 \\
\hline Por ende & 8 & 0.61 \\
\hline Ante eso & 10 & 0.77 \\
\hline Ante esto & 10 & 0.77 \\
\hline Es por eso que & 17 & 1.30 \\
\hline Fue así que & 19 & 1.45 \\
\hline En tanto & 21 & 1.61 \\
\hline Y eso & 36 & 2.75 \\
\hline Y así & 38 & 2.91 \\
\hline Entonces & 50 & 3.83 \\
\hline Así que & 199 & 15.23 \\
\hline Así & 403 & 30.83 \\
\hline Luego & 482 & 36.88 \\
\hline Total & 1307 & 100 \\
\hline & &
\end{tabular}

\section{Anexo 4. Conectores - Contra argumentativos}

\begin{tabular}{|l|r|r|}
\hline Marcadores & \multicolumn{1}{|c|}{$\mathbf{N}^{\circ}$} & \multicolumn{1}{|c|}{$\%$} \\
\hline En seguida & 1 & 0.04 \\
\hline A pesar que & 2 & 0.08 \\
\hline Y si bien & 3 & 0.12 \\
\hline Y aunque & 5 & 0.20 \\
\hline Es así que & 9 & 0.36 \\
\hline En cambio & 9 & 0.36 \\
\hline Por el contrario & 12 & 0.49 \\
\hline Y ahora & 14 & 0.57 \\
\hline Por el momento & 15 & 0.61 \\
\hline Fue así que & 19 & 0.77 \\
\hline Fue así que & 19 & 0.77 \\
\hline Mientras tanto & 20 & 0.81 \\
\hline A pesar de que & 22 & 0.89 \\
\hline Por ahora & 27 & 1.09 \\
\hline Eso sí & 56 & 2.27 \\
\hline Esta vez & 68 & 2.76 \\
\hline Si bien & 132 & 5.35 \\
\hline No obstante & 134 & 5.43 \\
\hline Aunque & 276 & 11.19 \\
\hline
\end{tabular}




\begin{tabular}{|l|r|r|}
\hline Ahora & 402 & 16.30 \\
\hline Pero & 560 & 22.70 \\
\hline Sin embargo & 662 & 26.83 \\
\hline Total & 2467 & 100.00 \\
\hline
\end{tabular}

Anexos 5. Reformuladores - Explicativos

\begin{tabular}{|l|r|r|}
\hline \multicolumn{1}{|c|}{ Marcadores } & Explicativos & \multicolumn{1}{c|}{$\mathbf{\%}$} \\
\hline De igual modo & 7 & 4.40 \\
\hline O sea & 20 & 12.58 \\
\hline Es decir & 36 & 22.64 \\
\hline Igual & 41 & 25.79 \\
\hline Esto es & 55 & 34.59 \\
\hline Total & 159 & 100.00 \\
\hline
\end{tabular}

Anexos 6. Reformuladores - Rectificativos

\begin{tabular}{|l|c|c|}
\hline Marcadores & Rectificativos & $\mathbf{\%}$ \\
\hline Más bien & 8 & 100 \\
\hline
\end{tabular}

\section{Anexos 7. Reformuladores - De distanciamiento}

\begin{tabular}{|l|r|r|}
\hline \multicolumn{1}{|c|}{ Marcadores } & Frecuencia & \multicolumn{1}{c|}{$\mathbf{\%}$} \\
\hline En todo caso & 1 & 6.25 \\
\hline En cualquier caso & 1 & 6.25 \\
\hline En cualquier caso & 1 & 6.25 \\
\hline De todas maneras & 4 & 25 \\
\hline De todos modos & 9 & 56.25 \\
\hline Total & 16 & 100 \\
\hline
\end{tabular}

Anexos 8. Reformuladores - Recapitulativos

\begin{tabular}{|l|r|r|}
\hline \multicolumn{1}{|c|}{ Tipo } & Frecuencia & \% \\
\hline En conclusión & 1 & 25 \\
\hline En fin & 3 & 75 \\
\hline Total & 4 & 100 \\
\hline
\end{tabular}

Anexos 9. Estructuradores - Comentadores

\begin{tabular}{|l|r|r|}
\hline \multicolumn{1}{|c|}{ Marcadores } & Comentadores & \multicolumn{1}{c|}{$\mathbf{\%}$} \\
\hline Pues & 9 & 4.5 \\
\hline Por otro lado & 189 & 94.5 \\
\hline Pues bien & 0 & 0 \\
\hline Así las cosas & 2 & 1 \\
\hline Total & 200 & 100 \\
\hline
\end{tabular}




\section{Anexos 10. Estructuradores - Digresores}

\begin{tabular}{|l|c|c|}
\hline \multicolumn{1}{|c|}{ Marcadores } & Digresores & $\mathbf{\%}$ \\
\hline A propósito & 10 & 100 \\
\hline Total & 10 & 100 \\
\hline
\end{tabular}

\section{Anexos 11. Estructuradores - Ordenadores}

\begin{tabular}{|l|r|r|}
\hline \multicolumn{1}{|c|}{ Marcadores } & Ordenadores & \multicolumn{1}{c|}{$\%$} \\
\hline Luego & 482 & 45.9 \\
\hline Finalmente & 182 & 17.3 \\
\hline Después & 162 & 15.4 \\
\hline De otro lado & 82 & 7.8 \\
\hline Primero & 71 & 6.8 \\
\hline Por último & 33 & 3.1 \\
\hline Segundo & 12 & 1.1 \\
\hline Por un lado & 9 & 0.9 \\
\hline En primer lugar & 7 & 0.7 \\
\hline Sobre el final & 6 & 0.6 \\
\hline Por otra parte & 2 & 0.2 \\
\hline por el lado & 2 & 0.2 \\
\hline Por una parte & 0 & 0 \\
\hline Total & 1050 & 100 \\
\hline
\end{tabular}

Anexo 12. Operadores argumentativos - De refuerzo argumentativo

\begin{tabular}{|c|c|c|}
\hline Marcadores & $\begin{array}{c}\text { De refuerzo } \\
\text { argumentativo }\end{array}$ & $\%$ \\
\hline En realidad & 12 & 41.38 \\
\hline En el fondo & 1 & 3.45 \\
\hline De hecho & 16 & 55.17 \\
\hline Total & 29 & 100 \\
\hline
\end{tabular}

Anexo 13. Operadores argumentativos - De concreción

\begin{tabular}{|l|r|r|}
\hline \multicolumn{1}{|c|}{ Marcadores } & De concreción & \% \\
\hline Por ejemplo & 14 & 93.33 \\
\hline En particular & 1 & 6.67 \\
\hline Total & 15 & 100 \\
\hline
\end{tabular}


Anexo 14. Marcadores conversacionales - de modalidad epistémica

\begin{tabular}{|l|r|r|}
\hline \multicolumn{1}{|c|}{ Marcadores } & \multicolumn{1}{|c|}{$\begin{array}{c}\text { De modalidad } \\
\text { epistémica }\end{array}$} & \multicolumn{1}{c|}{$\mathbf{\%}$} \\
\hline Efectivamente & 1 & 0.86 \\
\hline Desde luego & 3 & 2.59 \\
\hline Por lo visto & 4 & 3.45 \\
\hline Por lo que & 7 & 6.03 \\
\hline Por supuesto & 11 & 9.48 \\
\hline Claro & 35 & 30.17 \\
\hline Sin duda & 55 & 47.41 \\
\hline Total & 116 & 100 \\
\hline
\end{tabular}

Anexo 15. De modalidad deóntica

\begin{tabular}{|l|r|r|}
\hline Marcadores & $\begin{array}{c}\text { De modalidad } \\
\text { deóntica }\end{array}$ & $\mathbf{\%}$ \\
\hline Bien & 57 & 100 \\
\hline
\end{tabular}

Anexo 16. Enfocadores de la alteridad

\begin{tabular}{|l|r|c|}
\hline Marcadores & $\begin{array}{c}\text { Enfocadores de } \\
\text { la alteridad }\end{array}$ & $\mathbf{\%}$ \\
\hline Bueno & 153 & 100 \\
\hline
\end{tabular}

